\title{
Ruled and Rotational Surfaces Generated by Non-Null Curves with Zero Weighted Curvature in $\left(\mathbb{L}^{3}, a x^{2}+b y^{2}\right)$
}

\author{
Mustafa Altın*, Ahmet Kazan and H.Bayram Karadağ \\ (Communicated by Rafael López)
}

\begin{abstract}
In this study, firstly we give the weighted curvatures of non-null planar curves in LorentzMinkowski space with density $e^{a x^{2}+b y^{2}}$ and obtain the planar curves whose weighted curvatures vanish in this space under the condition that the constants $a$ and $b$ are not zero at the same time. After giving the Frenet vectors of the non-null planar curves with zero weighted curvature in Lorentz-Minkowski space with density $e^{a x^{2}}$, we create the Smarandache curves of them. With the aid of these curves and their Smarandache curves, we get the ruled surfaces whose base curves are non-null curves of which vanishing weighted curvature and ruling curves are Smarandache curves of them. Followingly, we give some characterizations for these ruled surfaces by obtaining the mean and Gaussian curvatures, distribution parameters and striction curves of them. Also, rotational surfaces which are generated by non-null planar curves with zero weighted curvatures in Lorentz-Minkowski space $E_{1}^{3}$ with density $e^{a x^{2}+b y^{2}}$ are studied under the condition that the constants $a$ and $b$ are not zero at the same time. We draw the graphics of the obtained surfaces.
\end{abstract}

Keywords: Weighted curvature; Lorentz-Minkowski space; spacelike and timelike curves; ruled surface; rotational surface.

AMS Subject Classification (2020): Primary: 53A35 ; Secondary: $53 B 30$.

\section{Introduction}

Since the curvature of a curve and the mean curvature of an $n$-dimensional hypersurface are important invariants for curves and surfaces, many authors have studied these notions for different types of curves and surfaces for a long time in different spaces, such as Euclidean, Minkowski, Galilean and pseudo-Galilean spaces ([2], [4], [9], [11], [14], [15], [19], [21] and etc.).

Here, we will study the planar curves, ruled surfaces and rotational surfaces in Lorentz-Minkowski space $\mathbb{L}^{3}$. So, let us recall some basic notions about them in this space.

Let $\mathbb{L}^{3}$ be the Lorentz-Minkowski 3-space endowed by Minkowski metric $g$ given by $g(. .)=.-\left(d x_{0}\right)^{2}+$ $\left(d x_{1}\right)^{2}+\left(d x_{2}\right)^{2}$, where $\left(x_{0}, x_{1}, x_{2}\right)$ are the canonical coordinates. Here, a vector field $X$ in $\mathbb{L}^{3}$ is called spacelike, timelike and lightlike(null) if $g(X, X)>0$ or $X=0, g(X, X)<0$ and $g(X, X)=0, X \neq 0$, respectively. The pseudonorm of a vector field $X$ is defined by $\|X\|=\sqrt{|g(X, X)|}$.

In $\mathbb{L}^{3}$, a curve $\alpha=\alpha(u)$ is spacelike, timelike or lightlike(null) if the velocity vector $\alpha^{\prime}(u)$ is spacelike, timelike or lightlike(null) for all $u \in I$, where $I$ is an open interval in $\mathbb{R}$ and $\alpha$ is a unit speed curve if $\left\|\alpha^{\prime}\right\|=1$.

Also, the Smarandache curves which play an important role in Smarandache geometry have been obtained with the aid of the Frenet frame of a curve. If we denote $T N$-Smarandache curve as $\beta_{T N}, T B-$ Smarandache curve as $\beta_{T B}, N B-$ Smarandache curve as $\beta_{N B}$ and $T N B-$ Smarandache curve as $\beta_{T N B}$ of a curve, then they 
are given by

$$
\begin{aligned}
& \beta_{T N}(u)=\frac{T(u)+N(u)}{\|T(u)+N(u)\|}, \beta_{T B}(u)=\frac{T(u)+B(u)}{\|T(u)+B(u)\|}, \\
& \beta_{N B}(u)=\frac{N(u)+B(u)}{\|N(u)+B(u)\|} \text { and } \beta_{T N B}(u)=\frac{T(u)+N(u)+B(u)}{\|T(u)+N(u)+B(u)\|},
\end{aligned}
$$

respectively. More information about Smarandache curves can be found in [1], [3], [25], [27] and etc.

On the other hand, the coefficients of the first and the second fundamental forms of a local parametrization of a spacelike or timelike surface

$$
\begin{aligned}
\Gamma: U \subset \mathbb{R}^{2} & \longrightarrow \mathbb{L}^{3} \\
(u, v) & \longrightarrow \Gamma(u, v),
\end{aligned}
$$

are

and

$$
E=g\left(\Gamma_{u}, \Gamma_{u}\right), F=g\left(\Gamma_{u}, \Gamma_{v}\right), G=g\left(\Gamma_{v}, \Gamma_{v}\right)
$$

$$
L=g\left(\mathcal{N}, \Gamma_{\mathrm{uu}}\right), M=g\left(\mathcal{N}, \Gamma_{\mathrm{u} v}\right), N=g\left(\mathcal{N}, \Gamma_{v v}\right),
$$

respectively. Here, $\mathcal{N}$ is the unit normal vector field of the surface given by

$$
\mathcal{N}=\frac{\Gamma_{u} \times \Gamma_{v}}{\left\|\Gamma_{u} \times \Gamma_{v}\right\|}
$$

and if we denote $\Omega=E G-F^{2}$, then the surface is spacelike when $\Omega>0$ and it is timelike when $\Omega<0$. Now, $g(\mathcal{N}, \mathcal{N})=\varepsilon$ and

$$
\left\|\Gamma_{u} \times \Gamma_{v}\right\|=\sqrt{-\varepsilon\left(E G-F^{2}\right)}=\sqrt{-\varepsilon \Omega} .
$$

Also, the mean curvature and Gaussian curvature of a surface are given by

$$
H=\varepsilon \frac{G L-2 F M+E N}{2\left(E G-F^{2}\right)} \text { and } K=\varepsilon \frac{L N-M^{2}}{E G-F^{2}},
$$

respectively (for detail, see [21]).

Furthermore, a ruled surface is a surface swept out by a straight line $l$ moving along a curve $\alpha(u)$.

The various positions of the generating line $l$ are called the rulings of the surface. This surface has a parametrization of

$$
\varphi(u, v)=\alpha(u)+v X(u), \quad u, v \in I \subset \mathbb{R},
$$

where $\alpha(u)$ is called the base curve and $X(u)$ is called the director vector of $l$. If the tangent plane is constant along a fixed ruling, then the ruled surface is called a developable surface. All other ruled surfaces are called skew surfaces. Also, we know that, a ruled surface is developable if and only if $c=0$ or $K=0$, where $T$ is the tangent vector of the base curve $\alpha, c=-\operatorname{det}\left(T, X, D_{T} X\right)$ and $K$ is Gaussian curvature of ruled surface.

The striction curve and distribution parameter of a ruled surface are

$$
\gamma(u)=\alpha(u)-\frac{g\left(\alpha^{\prime}(u), X^{\prime}(u)\right)}{\left\|X^{\prime}(u)\right\|^{2}} X(u)
$$

and

$$
\delta=\frac{\operatorname{det}\left[\alpha^{\prime}(u), X(u), X^{\prime}(u)\right]}{\left\|X^{\prime}(u)\right\|^{2}}
$$

respectively.

If $g\left(X^{\prime}(u), X^{\prime}(u)\right)=0$, then the ruled surface is a cylinder. The striction curve of the ruled surface, which is the cylinder, is $\gamma(u)=\alpha(u)$. For more details about ruled surfaces, we refer to [16], [26] and etc.

Moreover, a rotational surface in Lorentz-Minkowski space is generated by rotating of an arbitrary curve about an arbitrary axis. In this sense, the rotation matrices about the timelike axis $x_{1}=(1,0,0)$, spacelike axes $x_{2}=(0,1,0)$ and $x_{3}=(0,0,1)$ and lightlike line of plane $\left(x_{1} o x_{2}\right)$ spanned by the vector $(1,1,0)$ are

$$
\left(\begin{array}{ccc}
1 & 0 & 0 \\
0 & \cos v & -\sin v \\
0 & \sin v & \cos v
\end{array}\right),\left(\begin{array}{ccc}
\cosh v & 0 & \sinh v \\
0 & 1 & 0 \\
\sinh v & 0 & \cosh v
\end{array}\right),\left(\begin{array}{ccc}
\cosh v & \sinh v & 0 \\
\sinh v & \cosh v & 0 \\
0 & 0 & 1
\end{array}\right)
$$


and

$$
\left(\begin{array}{ccc}
1+\frac{v^{2}}{2} & -\frac{v^{2}}{2} & v \\
\frac{v^{2}}{2} & 1-\frac{v^{2}}{2} & v \\
v & -v & 1
\end{array}\right),
$$

respectively.

On the other hand, if $H$ is the mean curvature and $\eta$ is the normal vector field of an $n$-dimensional hypersurface; $\kappa$ is the curvature and $N$ is the normal vector of a curve, then the notions of weighted mean curvature of an $n$-dimensional hypersurface and weighted curvature of a curve on manifolds with density $e^{\varphi}$ have been introduced as

$$
H_{\varphi}=H-\frac{1}{n-1} \frac{d \varphi}{d \eta} \text { and } \kappa_{\varphi}=\kappa-\frac{d \varphi}{d N},
$$

respectively [17]. According to these definitions, some characterizations of weighted curves and surfaces in Euclidean, Minkowski and Galilean spaces with different densities have been studied by geometers, physicists and etc. (see [5]-[8], [10], [12], [18], [20], [22]-[24], [28]-[30] and etc.).

In the present paper, we will study the curves and surfaces in Lorentz-Minkowski space $\mathbb{L}^{3}$ with density $e^{a x^{2}+b y^{2}}$ and from now on, we will state the $\mathbb{L}^{3}$ with density $e^{a x^{2}+b y^{2}}$ as $\left(\mathbb{L}^{3}, a x^{2}+b y^{2}\right)$.

\section{Non-Null Curves with Zero Weighted Curvature in $\left(\mathbb{L}^{3}, a x^{2}+b y^{2}\right)$}

In this section, we give the weighted curvatures of spacelike and timelike planar curves in $\left(\mathbb{L}^{3}, a x^{2}+b y^{2}\right)$ and obtain the curves whose weighted curvatures are zero in $\left(\mathbb{L}^{3}, a x^{2}+b y^{2}\right)$ according to the cases of constants $a$ and $b$.

\subsection{Spacelike Curves with Zero Weighted Curvature in $\left(\mathbb{L}^{3}, a x^{2}+b y^{2}\right)$}

Let us suppose that $\alpha$ is a spacelike curve in Lorentz-Minkowski space $\mathbb{L}^{3}$.

One can easily see that, the Frenet vectors and curvature of a spacelike planar curve $\alpha(u)=(x(u), y(u), 0)$ in the (xoy)-plane are

$$
\begin{aligned}
T & =\frac{1}{\sqrt{y^{\prime}(u)^{2}-x^{\prime}(u)^{2}}}\left(x^{\prime}(u), y^{\prime}(u), 0\right), \\
N & =\frac{1}{\sqrt{y^{\prime}(u)^{2}-x^{\prime}(u)^{2}}}\left(-y^{\prime}(u),-x^{\prime}(u), 0\right), \\
B & =(0,0,1) \\
\kappa & =\frac{x^{\prime \prime}(u) y^{\prime}(u)-x^{\prime}(u) y^{\prime \prime}(u)}{\left(y^{\prime}(u)^{2}-x^{\prime}(u)^{2}\right)^{3 / 2}} .
\end{aligned}
$$

Using (2.1) in (1.12), the weighted curvature $\kappa_{\varphi}(u)$ of the spacelike planar curve $\alpha(u)=(x(u), y(u), 0)$ in $\left(\mathbb{L}^{3}, a x^{2}+b y^{2}\right)$ is obtained as

$$
\kappa_{\varphi}(u)=\frac{x^{\prime \prime}(u) y^{\prime}(u)-x^{\prime}(u) y^{\prime \prime}(u)-\left(y^{\prime}(u)^{2}-x^{\prime}(u)^{2}\right)\left(2 a x(u) y^{\prime}(u)-2 b y(u) x^{\prime}(u)\right)}{\left(y^{\prime}(u)^{2}-x^{\prime}(u)^{2}\right)^{3 / 2}} .
$$

Hence, we have

Proposition 2.1. The weighted curvature $\kappa_{\varphi}(u)$ of a spacelike planar curve $\alpha(u)=(x(u), y(u), 0)$ in $\left(\mathbb{L}^{3}, a x^{2}+b y^{2}\right)$ is zero iff

$$
x^{\prime}(u) y^{\prime \prime}(u)+2 a x(u) y^{\prime}(u)\left(y^{\prime}(u)^{2}-x^{\prime}(u)^{2}\right)=x^{\prime \prime}(u) y^{\prime}(u)+2 b y(u) x^{\prime}(u)\left(y^{\prime}(u)^{2}-x^{\prime}(u)^{2}\right) .
$$

Now, we will investigate the weighted curvature $\kappa_{\varphi}(u)$ of a spacelike planar curve $\alpha(u)$ in $\left(\mathbb{L}^{3}, a x^{2}+b y^{2}\right)$ for different values of not all zero constants $a$ and $b$.

Firstly, let us assume that $a \neq 0, b=0$.

In this case, from (2.2) we have

$$
\kappa_{\varphi}(u)=\frac{x^{\prime \prime}(u) y^{\prime}(u)-x^{\prime}(u) y^{\prime \prime}(u)-2 a x(u) y^{\prime}(u)\left(y^{\prime}(u)^{2}-x^{\prime}(u)^{2}\right)}{\left(y^{\prime}(u)^{2}-x^{\prime}(u)^{2}\right)^{3 / 2}} .
$$


So,

Proposition 2.2. The weighted curvature $\kappa_{\varphi}(u)$ of a spacelike planar curve $\alpha(u)=(x(u), y(u), 0)$ in $\left(\mathbb{L}^{3}\right.$, ax $\left.x^{2}\right)$ is zero iff

$$
x^{\prime}(u) y^{\prime \prime}(u)+2 a x(u) y^{\prime}(u)\left(y^{\prime}(u)^{2}-x^{\prime}(u)^{2}\right)=x^{\prime \prime}(u) y^{\prime}(u) .
$$

Here, if $x^{\prime}(u)=0$, then from (2.3), we have $y^{\prime}(u)=0$ or $x(u)=0$.

If $x^{\prime}(u) \neq 0$, then

$$
y(u)=c_{2} \pm \int_{1}^{u} \frac{x^{\prime}(t) e^{a x^{2}(t)}}{\sqrt{e^{2 a x^{2}(t)}+c_{1}}} d t
$$

is a solution for the equation (2.3), where $c_{1}, c_{2} \in \mathbb{R}, c_{1}<0$ and $e^{2 a x(u)}+c_{1}>0$. Thus, we have

Theorem 2.1. The spacelike planar curve with zero weighted curvature in $\left(\mathbb{L}^{3}\right.$, ax $\left.x^{2}\right)$ can be parametrized by

$$
\alpha_{1}(u)=(0, y(u), 0)
$$

or

$$
\alpha_{2}(u)=\left(x(u), c_{2} \pm \int_{1}^{u} \frac{x^{\prime}(t) e^{a x^{2}(t)}}{\sqrt{e^{2 a x^{2}(t)}+c_{1}}} d t, 0\right),
$$

where $c_{1}, c_{2} \in \mathbb{R}, c_{1}<0$ and $e^{2 a x(u)}+c_{1}>0$.

Now, after giving the Frenet vectors of the spacelike curve (2.4) (taking " \pm " in (2.4) as "+") with zero weighted curvature in $\left(\mathbb{L}^{3}, a x^{2}\right)$, we will create the Smarandache curves of it. The Frenet vectors of the spacelike curve (2.4) are given by

$$
\begin{aligned}
T & =\left(\frac{\sqrt{e^{2 a x^{2}(u)}+c_{1}}}{\sqrt{-c_{1}}}, \frac{e^{a x^{2}(u)}}{\sqrt{-c_{1}}}, 0\right), \\
N & =\left(\frac{-e^{a x^{2}(u)}}{\sqrt{-c_{1}}}, \frac{-\sqrt{e^{2 a x^{2}(u)}+c_{1}}}{\sqrt{-c_{1}}}, 0\right), \\
B & =(0,0,1),
\end{aligned}
$$

where $c_{1}<0$ and $e^{2 a x^{2}(u)}+c_{1}>0$. From (2.5), we have $\|T+N\|=\|N+B\|=0$ and so we consider only the $T B$ and $T N B$ Smarandache curves of the spacelike curve (2.4). From (2.5), the $T B$ Smarandache curve $S_{1 T B}$ and the $T N B$ Smarandache curve $S_{1 T N B}$ of the spacelike curve (2.4) with zero weighted curvature in $\left(\mathbb{L}^{3}, a x^{2}\right)$ are obtained as

$$
S_{1 T B}(u)=\left(\frac{\sqrt{e^{2 a x^{2}(u)}+c_{1}}}{\sqrt{-2 c_{1}}}, \frac{e^{a x^{2}(u)}}{\sqrt{-2 c_{1}}}, \frac{1}{\sqrt{2}}\right)
$$

and

$$
S_{1 T N B}(u)=\left(\frac{\sqrt{e^{2 a x^{2}(u)}+c_{1}}-e^{a x^{2}(u)}}{\sqrt{-c_{1}}}, \frac{e^{a x^{2}(u)}-\sqrt{e^{2 a x^{2}(u)}+c_{1}}}{\sqrt{-c_{1}}}, 1\right),
$$

respectively.

Now, if $b \neq 0, a=0$, then from (2.2), the weighted curvature $\kappa_{\varphi}(u)$ of a spacelike planar curve $\alpha(u)=$ $(x(u), y(u), 0)$ in $\left(\mathbb{L}^{3}, b y^{2}\right)$ is

$$
\kappa_{\varphi}(u)=\frac{x^{\prime \prime}(u) y^{\prime}(u)-x^{\prime}(u) y^{\prime \prime}(u)+2 b y(u) x^{\prime}(u)\left(y^{\prime}(u)^{2}-x^{\prime}(u)^{2}\right)}{\left(y^{\prime}(u)^{2}-x^{\prime}(u)^{2}\right)^{3 / 2}} .
$$

So,

Proposition 2.3. The weighted curvature $\kappa_{\varphi}(u)$ of a spacelike planar curve $\alpha(u)=(x(u), y(u), 0)$ in $\left(\mathbb{L}^{3}, b^{2}\right)$ is zero iff

$$
x^{\prime}(u) y^{\prime \prime}(u)=x^{\prime \prime}(u) y^{\prime}(u)+2 b y(u) x^{\prime}(u)\left(y^{\prime}(u)^{2}-x^{\prime}(u)^{2}\right) .
$$


If $x^{\prime}(u)=0$, then from $(2.8)$, we have

$$
\alpha(u)=\left(c_{3}, y(u), 0\right), c_{3} \in \mathbb{R} .
$$

If $x^{\prime}(u) \neq 0$, again from $(2.8)$, we get

$$
\alpha(u)=\left(c_{4} \pm \int_{1}^{u} \frac{y^{\prime}(t)}{\sqrt{c_{5} e^{2 b y^{2}(t)}+1}} d t, y(u), 0\right),
$$

where $c_{4}, c_{5} \in \mathbb{R}$ and $c_{5}>0$. So, we obtain

Theorem 2.2. The spacelike planar curve with zero weighted curvature in $\left(\mathbb{L}^{3}, b y^{2}\right)$ can be parametrized by

$$
\alpha_{3}(u)=\left(c_{3}, y(u), 0\right)
$$

or

$$
\alpha_{4}(u)=\left(c_{4} \pm \int_{1}^{u} \frac{y^{\prime}(t)}{\sqrt{c_{5} e^{2 b y^{2}(t)}+1}} d t, y(u), 0\right)
$$

where $c_{3}, c_{4}, c_{5} \in \mathbb{R}$ and $c_{5}>0$.

\subsection{Timelike Curves with Zero Weighted Curvature in $\left(\mathbb{L}^{3}, a x^{2}+b y^{2}\right)$}

Let $\alpha$ be a timelike planar curve in Lorentz-Minkowski space.

Now, the Frenet vectors and curvature of a timelike planar curve $\alpha(u)=(x(u), y(u), 0)$ in the (xoy)-plane are

$$
\begin{aligned}
T & =\frac{1}{\sqrt{x^{\prime}(u)^{2}-y^{\prime}(u)^{2}}}\left(x^{\prime}(u), y^{\prime}(u), 0\right), \\
N & =\frac{1}{\sqrt{x^{\prime}(u)^{2}-y^{\prime}(u)^{2}}}\left(y^{\prime}(u), x^{\prime}(u), 0\right), \\
B & =(0,0,1), \\
\kappa & =\frac{x^{\prime}(u) y^{\prime \prime}(u)-x^{\prime \prime}(u) y^{\prime}(u)}{\left(x^{\prime}(u)^{2}-y^{\prime}(u)^{2}\right)^{3 / 2}} .
\end{aligned}
$$

If we use (2.10) in (1.12), then the weighted curvature $\kappa_{\varphi}(u)$ of a timelike planar curve $\alpha(u)=(x(u), y(u), 0)$ in $\left(\mathbb{L}^{3}, a x^{2}+b y^{2}\right)$ is

$$
\kappa_{\varphi}=\frac{x^{\prime}(u) y^{\prime \prime}(u)-x^{\prime \prime}(u) y^{\prime}(u)-\left(x^{\prime}(u)^{2}-y^{\prime}(u)^{2}\right)\left(-2 a x(u) y^{\prime}(u)+2 b y(u) x^{\prime}(u)\right)}{\left(x^{\prime}(u)^{2}-y^{\prime}(u)^{2}\right)^{3 / 2}} .
$$

By using the same procedure in the above subsection, we obtain

Theorem 2.3. The timelike planar curve with zero weighted curvature in $\left(\mathbb{L}^{3}, a x^{2}\right)$ and $\left(\mathbb{L}^{3}, b y^{2}\right)$ can be parametrized by

$$
\begin{aligned}
& \alpha_{5}(u)=\left(x(u), c_{6}, 0\right), \\
& \alpha_{6}(u)=\left(x(u), c_{7} \pm \int_{1}^{u} \frac{x^{\prime}(t)}{\sqrt{c_{8} e^{2 a x^{2}(t)}+1}} d t, 0\right)
\end{aligned}
$$

and

$$
\begin{aligned}
& \alpha_{7}(u)=(x(u), 0,0), \\
& \alpha_{8}(u)=\left(c_{9} \pm \int_{1}^{u} \frac{e^{b y^{2}(t)} y^{\prime}(t)}{\sqrt{e^{2 b y^{2}(t)}+c_{10}}} d t, y(u), 0\right),
\end{aligned}
$$

respectively, where $c_{7}, c_{8}, c_{9}, c_{10} \in \mathbb{R}, c_{8}>0, c_{10}<0$ and $e^{2 b y^{2}(u)}+c_{10}>0$. 
Now, after giving the Frenet vectors of the timelike curve (2.12) (taking " \pm " in (2.12) as "+") with zero weighted curvature in $\left(\mathbb{L}^{3}, a x^{2}\right)$, we will create its Smarandache curves. The Frenet vectors of the timelike curve (2.12) are given by

$$
\begin{aligned}
T & =\left(\frac{\sqrt{c_{8} e^{2 a x^{2}(u)}+1}}{\sqrt{c_{8} e^{2 a x^{2}(u)}}}, \frac{1}{\sqrt{c_{8} e^{2 a x^{2}(u)}}}, 0\right), \\
N & =\left(\frac{1}{\sqrt{c_{8} e^{2 a x^{2}(u)}}}, \frac{\sqrt{c_{8} e^{2 a x^{2}(u)}+1}}{\sqrt{c_{8} e^{2 a x^{2}(u)}}}, 0\right), \\
B & =(0,0,1),
\end{aligned}
$$

where $c_{8}>0$. From (2.14), we have $\|T+N\|=\|T+B\|=0$ and so we consider only the $N B$ and $T N B$ Smarandache curves of the timelike curve (2.12). From (2.14), the $N B$ Smarandache curve $S_{2 N B}$ and the $T N B$ Smarandache curve $S_{2 T N B}$ of the timelike curve (2.12) with zero weighted curvature in $\left(\mathbb{L}^{3}, a x^{2}\right)$ are obtained as follows

$$
S_{2 N B}(u)=\left(\frac{1}{\sqrt{c_{8} e^{2 a x^{2}(u)}}}, \frac{\sqrt{c_{8} e^{2 a x^{2}(u)}+1}}{\sqrt{c_{8} e^{2 a x^{2}(u)}}}, \frac{1}{\sqrt{2}}\right)
$$

and

$$
S_{2 T N B}(u)=\left(\frac{1+\sqrt{c_{8} e^{2 a x^{2}(u)}+1}}{\sqrt{c_{8} e^{2 a x^{2}(u)}}}, \frac{1+\sqrt{c_{8} e^{2 a x^{2}(u)}+1}}{\sqrt{c_{8} e^{2 a x^{2}(u)}}}, 1\right)
$$

respectively.

Figure 1 (a) shows the curve (2.4) for $x(u)=\sqrt{\ln \left(u^{2}\right)}, c_{1}=-0.1, c_{2}=4, a=1$ and Figure 1 (b) shows the curve (2.12) for $y(u)=\sqrt{\ln (u)}, c_{7}=4$ and $c_{8}=a=1$.

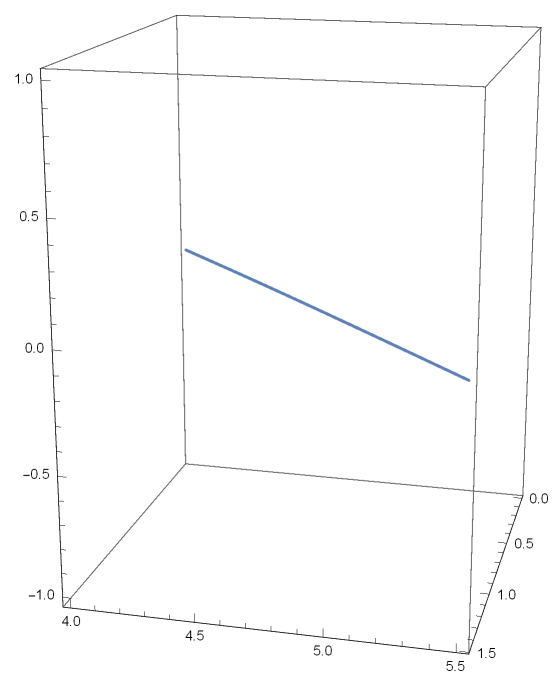

(a) Curve (2.4)



(b) Curve (2.12)

Figure 1

\section{Ruled Surfaces Generated by Non-Null Curves with Vanishing Weighted Curvature in} $\left(\mathbb{L}^{3}, a x^{2}+b y^{2}\right)$

In this section, we obtain the ruled surfaces generated by spacelike and timelike curves with vanishing weighted curvatures in $\left(\mathbb{L}^{3}, a x^{2}\right)$ and $\left(\mathbb{L}^{3}, b y^{2}\right)$. We get the mean and Gaussian curvatures of these surfaces, give some characterizations for them and draw the graphics of these surfaces. 


\subsection{Ruled Surfaces whose Base Curves are (2.4) and Ruling Curves are Smarandache Curves of (2.4)}

In this subsection, firstly we obtain the ruled surfaces generated by spacelike planar curve (2.4) with zero weighted curvature in $\left(\mathbb{L}^{3}, a x^{2}\right)$ and its Smarandache curves (2.6) and (2.7). Then, we give some characterizations for these ruled surfaces by obtaining the mean and Gaussian curvatures, distribution parameter and striction curve of them. Finally, we draw the graphics of these surfaces.

Throughout this subsection, the base curves of the ruled surfaces will be taken as the curve (2.4).

First, let the ruling curve of the ruled surface be the $T B$-Smarandache curve $S_{1 T B}(u)$ of the curve (2.4). Thus, from (1.7), (2.4) and (2.6), the ruled surface $\varphi_{1 T B}(u, v)$ in Lorentz-Minkowski space can be parametrized by

$$
\begin{aligned}
\varphi_{1 T B}(u, v) & =\alpha_{2}(u)+v S_{1 T B}(u) \\
& =\left(x(u)+\frac{v \sqrt{e^{2 a x^{2}(u)}+c_{1}}}{\sqrt{-2 c_{1}}}, c_{2}+\int_{1}^{u} \frac{e^{a x^{2}(t)} x^{\prime}(t)}{\sqrt{e^{2 a x^{2}(t)}+c_{1}}} d t+\frac{v e^{a x^{2}(u)}}{\sqrt{-2 c_{1}}}, \frac{v}{\sqrt{2}}\right) .
\end{aligned}
$$

where $c_{1}<0, e^{2 a x^{2}(t)}+c_{1}>0$ and $e^{2 a x^{2}(u)}+c_{1}>0$.

The normal vector of this surface is

$$
\begin{gathered}
\mathcal{N}_{1 T B}(u, v)=\frac{1}{\sqrt{4 a^{2} v^{2} x^{2}(u) e^{2 a x^{2}(u)}+c_{1}}}\left(\frac{-e^{a x^{2}(u)}\left(\sqrt{-c_{1}}+\sqrt{2} a v x(u) \sqrt{e^{2 a x^{2}(u)}+c_{1}}\right)}{\sqrt{-c_{1}}},\right. \\
-\frac{\sqrt{-c_{1}} \sqrt{e^{2 a x^{2}(u)}+c_{1}}+\sqrt{2} a v x(u) e^{2 a x^{2}(u)}}{\sqrt{-c_{1}}}, \\
\left.\sqrt{2} \operatorname{avx}(u) e^{a x^{2}(u)}\right) .
\end{gathered}
$$

From (3.2), we have $g\left(N_{1 T B}, N_{1 T B}\right)=\varepsilon=1$ and so, the surface is timelike.

Thus, the Gaussian and mean curvatures of (3.1) are obtained by

$$
K=-\frac{4 c_{1} a^{2} x^{2}(u) e^{2 a x^{2}(u)}}{\left(c_{1}+4 a^{2} x^{2}(u) v^{2} e^{2 a x^{2}(u)}\right)^{2}}
$$

and

$$
H=\frac{-\sqrt{-2 c_{1}} a \sqrt{e^{2 a x^{2}(u)}+c_{1}} v e^{a x^{2}(u)}\left(1+2 a x^{2}(u)\right)+4 a^{3} x^{3}(u) v^{2} e^{3 a x^{2}(u)}}{2\left(c_{1}+4 a^{2} x^{2}(u) v^{2} e^{2 a x^{2}(u)}\right)^{3 / 2}},
$$

respectively.

Theorem 3.1. The ruled surface (3.1) generated by spacelike planar curve (2.4) with zero weighted curvature in $\left(\mathbb{L}^{3}\right.$, ax $\left.x^{2}\right)$ is not flat and minimal. But the mean curvature of (3.1) vanishes for $v=0$.

Also from (1.9), the distribution parameter of $\varphi_{1 T B}(u, v)$ is

$$
\delta_{1 T B}=\frac{2 e^{2 a x^{2}(u)}+c_{1}}{2 \sqrt{-c_{1}} a x(u) e^{a x^{2}(u)} \sqrt{\left(c_{2}+\int_{1}^{u} \frac{e^{a x^{2}(t)} x^{\prime}(t)}{\sqrt{e^{2 a x^{2}(t)}+c_{1}}} d t\right)^{2}-x^{2}(u)}} .
$$

So, we have

Theorem 3.2. The ruled surface (3.1) generated by spacelike planar curve (2.4) with zero weighted curvature in $\left(\mathbb{L}^{3}\right.$, ax $\left.x^{2}\right)$ is not developable.

From (1.8), the parametrization of the striction curve $\gamma_{1 T B}(u)$ on $\varphi_{1 T B}(u, v)$ is

$$
\gamma_{1 T B}(u)=\alpha_{2}(u) .
$$

Thus, 
Theorem 3.3. The base curve and the striction curve of (3.1) generated by spacelike planar curve (2.4) with zero weighted curvature in $\left(\mathbb{L}^{3}, a x^{2}\right)$ coincide.

Figure 2 shows the ruled surface (3.1) for $x(u)=\sqrt{\ln (u)}, c_{1}=-0.1, c_{2}=4$ and $a=1$.

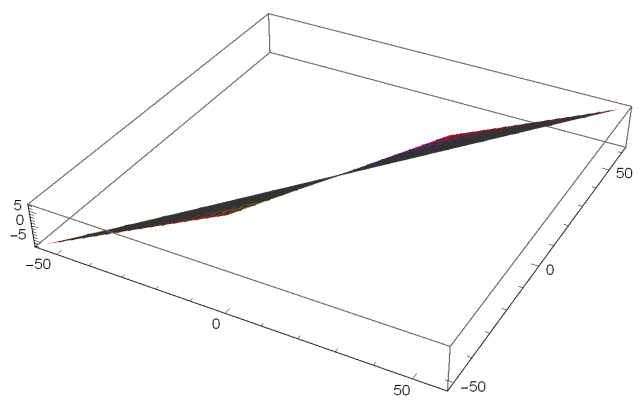

Figure 2

Now, let the ruling curve of the ruled surface be the $T N B$-Smarandache curve $S_{1 T N B}(u)$ of the curve (2.4). Then from (1.7), (2.4) and (2.7), the ruled surface $\varphi_{1 T N B}(u, v)$ in Lorentz-Minkowski space can be parametrized by

$$
\begin{aligned}
\varphi_{1 T N B}(u, v)= & \alpha_{2}(u)+v S_{1 T N B}(u) \\
= & \left(x(u)+\frac{v\left(\sqrt{e^{2 a x^{2}(u)}+c_{1}}-e^{a x^{2}(u)}\right)}{\sqrt{-c_{1}}},\right. \\
& \left.c_{2}+\int_{1}^{u} \frac{e^{a x^{2}(t)} x^{\prime}(t)}{\sqrt{e^{2 a x^{2}(t)}+c_{1}}} d t+\frac{v\left(e^{a x^{2}(u)}-\sqrt{e^{2 a x^{2}(u)}+c_{1}}\right)}{\sqrt{-c_{1}}}, v\right) .
\end{aligned}
$$

where $c_{1}<0, e^{2 a x^{2}(t)}+c_{1}>0$ and $e^{2 a x^{2}(u)}+c_{1}>0$.

The normal vector of this surface is

$$
\begin{aligned}
\mathcal{N}_{1 T N B}= & \left(\frac{-a v x(u) e^{2 a x^{2}(u)}\left(\sqrt{-c_{1}}+2 a v x(u)\left(\sqrt{e^{2 a x^{2}(u)}+c_{1}}-e^{a x^{2}(u)}\right)\right)}{2\left(\sqrt{-c_{1}} a v x(u) e^{a x^{2}(u)}\right)^{3 / 2}},\right. \\
& \frac{2 a v x(u) e^{a x^{2}(u)}\left(\sqrt{e^{2 a x^{2}(u)}+c_{1}}-e^{a x^{2}(u)}\right)-\sqrt{-c_{1}} \sqrt{e^{2 a x^{2}(u)}+c_{1}}}{\sqrt{2} \sqrt{-c_{1}} \sqrt{\sqrt{-c_{1}} a v x(u) e^{a x(u)}}}, \\
& \left.\frac{\sqrt{-c_{1}}}{2 \sqrt{\sqrt{-c_{1}} a x(u) v e^{a x^{2}(u)}}}\right) .
\end{aligned}
$$

From (3.8), we have $g\left(N_{1 T N B}, N_{1 T N B}\right)=\varepsilon=1$; that is, the surface is timelike.

So, the Gaussian and mean curvatures of (3.7) are obtained by

$$
K=\frac{1}{4 v^{2}}
$$

and

$$
H=-\frac{a e^{a x^{2}(u)}\left(\sqrt{-c_{1}} v \sqrt{e^{2 a x^{2}(u)}+c_{1}}+c_{1} x(u)+2 \sqrt{-c_{1}} a x^{2}(u) v\left(\sqrt{e^{2 a x^{2}(u)}+c_{1}}-2 e^{a x^{2}(u)}\right)\right)}{8\left(\sqrt{-c_{1}} a v x(u) e^{a x^{2}(u)}\right)},
$$

respectively. Hence, 
Theorem 3.4. The ruled surface (3.7) generated by spacelike planar curve (2.4) with zero weighted curvature in $\left(\mathbb{L}^{3}, a x^{2}\right)$ is not flat and minimal.

Since $g\left(S_{1 T N B}^{\prime}(u), S_{1 T N B}^{\prime}(u)\right)=0$, the ruled surface $\varphi_{1 T N B}(u, v)$ in Lorentz-Minkowski space is cylinder.

From (1.8), the parametrization of the striction curve $\gamma_{1 T N B}(u)$ on $\varphi_{1 T N B}(u, v)$ is

$$
\gamma_{1 T N B}(u)=\alpha_{2}(u) .
$$

Theorem 3.5. The base curve and the striction curve of (3.7) generated by spacelike planar curve (2.4) with zero weighted curvature in $\left(\mathbb{L}^{3}, a x^{2}\right)$ coincide.

Figure 3 shows the ruled surface (3.7) for $x(u)=\sqrt{\ln (u)}, c_{1}=-0.1, c_{2}=4$ and $a=1$.

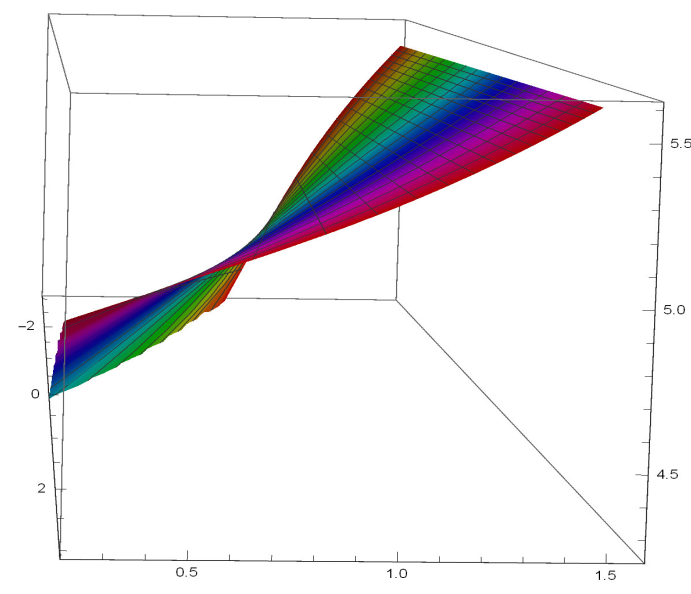

Figure 3

One can obtain the similar results for ruled surfaces whose base curves are timelike planar curve (2.13) with zero weighted curvature in $\left(\mathbb{L}^{3}, b y^{2}\right)$ and ruling curves are Smarandache curves of (2.13).

\subsection{Ruled Surfaces whose Base Curves are (2.12) and Ruling Curves are Smarandache Curves of (2.12)}

In this subsection, firstly we obtain the ruled surfaces generated by timelike planar curve (2.12) with zero weighted curvature in $\left(\mathbb{L}^{3}, a x^{2}\right)$ and its Smarandache curves (2.15) and (2.16). Then, we give some characterizations for these ruled surfaces by obtaining the mean and Gaussian curvatures, distribution parameter and striction curve of them. Finally, we draw the graphics of these surfaces.

Throughout this subsection, the base curves of the ruled surfaces will be taken as the curve (2.12).

First, let the ruling curve of the ruled surface be the $N B$-Smarandache curve $S_{2 N B}(u)$ of the curve (2.12). Then, from (1.7), (2.12) and (2.15), the ruled surface $\varphi_{2 N B}(u, v)$ in Lorentz-Minkowski space can be parametrized by

$$
\begin{aligned}
\varphi_{2 N B}(u, v)= & \alpha_{6}(u)+v S_{2 N B}(u) \\
= & \left(x(u)+\frac{v}{\sqrt{2 c_{8} e^{2 a x^{2}(u)}}},\right. \\
& \left.c_{7}+\int_{1}^{u} \frac{x^{\prime}(t)}{\sqrt{c_{8} e^{2 a x^{2}(t)}+1}} d t+\frac{v \sqrt{c_{8} e^{2 a x^{2}(u)}+1}}{\sqrt{2 c_{8} e^{2 a x^{2}(u)}}}, \frac{v}{\sqrt{2}}\right),
\end{aligned}
$$

where $c_{8}>0$.

The normal vector of (3.12) is

$$
\mathcal{N}_{2 N B}=\left(\frac{1}{\sqrt{2 c_{8} e^{2 a x^{2}(u)}}}, \frac{\sqrt{c_{8} e^{2 a x^{2}(u)}+1}}{\sqrt{2 c_{8} e^{2 a x^{2}(u)}}}, \frac{1}{\sqrt{2}}\right) .
$$


From (3.13), we have $g\left(\mathcal{N}_{2 N B}, \mathcal{N}_{2 N B}\right)=\varepsilon=1$ and so, the surface is timelike.

Thus, the Gaussian and mean curvatures of this surface are obtained as

$$
K=0
$$

and

$$
H=\frac{-a x(u)}{\sqrt{2\left(c_{8} e^{2 a x^{2}(u)}-2 a v x(u) \sqrt{2 c_{8} e^{2 a x^{2}(u)}}+2(\operatorname{avx}(u))^{2}\right)}},
$$

respectively.

Theorem 3.6. The ruled surface (3.12) generated by timelike planar curve (2.12) with zero weighted curvature in $\left(\mathbb{L}^{3}, a x^{2}\right)$ is flat and not minimal. But the mean curvature of the ruled surface (3.12) vanishes for $x(u)=0$.

From (3.14), we have

Theorem 3.7. The ruled surface (3.12) generated by timelike planar curve (2.12) with zero weighted curvature in $\left(\mathbb{L}^{3}, a x^{2}\right)$ is developable.

From (1.8), the parametrization of the striction curve $\gamma_{2 N B}(u)$ on $\varphi_{2 N B}(u, v)$ is

$$
\gamma_{2 N B}(u)=\left(\alpha_{6}(u)+\frac{\sqrt{c_{8} e^{2 a x^{2}(u)}}}{\sqrt{2} a x(u) \sqrt{\left(c_{7}+\int_{1}^{u} \frac{x^{\prime}(t)}{\sqrt{c_{8} e^{2 a x^{2}(t)}+1}} d t\right)^{2}-x^{2}(u)}}\right) S_{2 N B}(u) .
$$

Thus,

Theorem 3.8. The base curve and the striction curve of (3.12) generated by timelike planar curve (2.12) with zero weighted curvature in $\left(\mathbb{L}^{3}, a x^{2}\right)$ never coincide.

Figure 4 shows the ruled surface (3.12) for $x(u)=\sqrt{\ln (u)}, c_{7}=c_{8}=4$ and $a=1$.



Figure 4

Now, let the ruling curve of the ruled surface be the $T N B$-Smarandache curve $S_{2 T N B}(u)$ of the curve (2.12). Then from (1.7), (2.12) and (2.16), the ruled surface $\varphi_{2 T N B}(u, v)$ in Lorentz-Minkowski space can be 
parametrized by

$$
\begin{aligned}
\varphi_{2 T N B}(u, v)= & \alpha_{6}(u)+v S_{2 T N B}(u) \\
= & \left(x(u)+\frac{v\left(\sqrt{c_{8} e^{2 a x^{2}(u)}+1}+1\right)}{\sqrt{c_{8} e^{2 a x^{2}(u)}}},\right. \\
& \left.c_{7}+\int_{1}^{u} \frac{x^{\prime}(t)}{\sqrt{c_{8} e^{2 a x^{2}(t)}+1}} d t+\frac{v\left(\sqrt{c_{8} e^{2 a x^{2}(u)}+1}+1\right)}{\sqrt{c_{8} e^{2 a x^{2}(u)}}}, v\right) .
\end{aligned}
$$

The normal vector of this surface is

$$
\begin{aligned}
\mathcal{N}_{2 T N B} & =\left(\frac{2 a v x(u)\left(1+\sqrt{c_{8} e^{2 a x^{2}(u)}+1}\right)-\sqrt{c_{8} e^{2 a x^{2}(u)}}}{\left.\sqrt{2 c_{8} e^{2 a x^{2}(u)}\left(c_{8} e^{2 a x^{2}(u)}-2 a v x(u) \sqrt{c_{8} e^{2 a x^{2}(u)}}\right.}\right)},\right. \\
& \frac{2 a v x(u)\left(1+\sqrt{c_{8} e^{2 a x^{2}(u)}}\right)-\sqrt{\left(c_{8} e^{2 a x^{2}(u)}+1\right) c_{8} e^{2 a x^{2}(u)}}}{\left.\sqrt{2 c_{8} e^{2 a x^{2}(u)}\left(c_{8} e^{2 a x^{2}(u)}-2 a v x(u) \sqrt{c_{8} e^{2 a x^{2}(u)}}\right.}\right)}, \\
& \frac{\sqrt{c_{8} e^{2 a x^{2}(u)}}}{\left.\sqrt{2\left(c_{8} e^{2 a x^{2}(u)}-2 a v x(u) \sqrt{c_{8} e^{2 a x^{2}(u)}}\right.}\right)} .
\end{aligned}
$$

From (3.18), we have $g\left(\mathcal{N}_{2 T N B}, \mathcal{N}_{2 T N B}\right)=\varepsilon=1$; that is, the surface is timelike.

So, the Gaussian and mean curvatures of this surface are obtained as

$$
K=\frac{(a x(u))^{2}}{c_{8} e^{2 a x^{2}(u)}-4 a v x(u) \sqrt{c_{8} e^{2 a x^{2}(u)}}+(2 a v x(u))^{2}}
$$

and

$$
H=\frac{2 a v x^{2}(u)\left(2+\sqrt{c_{8} e^{2 a x^{2}(u)}+1}\right)-v \sqrt{c_{8} e^{2 a x^{2}(u)}+1}-3 x(u) \sqrt{c_{8} e^{2 a x^{2}(u)}}}{2\left(\sqrt{c_{8} e^{2 a x^{2}(u)}}-2 a v x(u)\right) \sqrt{2\left(c_{8} e^{2 a x^{2}(u)}-2 a v x(u) \sqrt{c_{8} e^{2 a x^{2}(u)}}\right)}}
$$

respectively. Hence,

Theorem 3.9. The ruled surface (3.17) generated by timelike planar curve (2.12) with zero weighted curvature in $\left(\mathbb{L}^{3}, a x^{2}\right)$ is not flat and minimal.

Since $g\left(S_{2 T N B}^{\prime}(u), S_{2 T N B}^{\prime}(u)\right)=0$, the ruled surface $\varphi_{2 T N B}(u, v)$ in Lorentz-Minkowski space is cylinder.

From (1.8), the parametrization of the striction curve $\gamma_{2 T N B}(u)$ on $\varphi_{2 T N B}(u, v)$ is

$$
\gamma_{2 T N B}(u)=\alpha_{6}(u) .
$$

Theorem 3.10. The base curve and the striction curve of (3.17) generated by timelike planar curve (2.12) with zero weighted curvature in $\left(\mathbb{L}^{3}, a x^{2}\right)$ coincide.

Figure 5 shows the ruled surface (3.17) for $x(u)=\sqrt{\ln (u)}, c_{7}=c_{8}=4$ and $a=1$. 


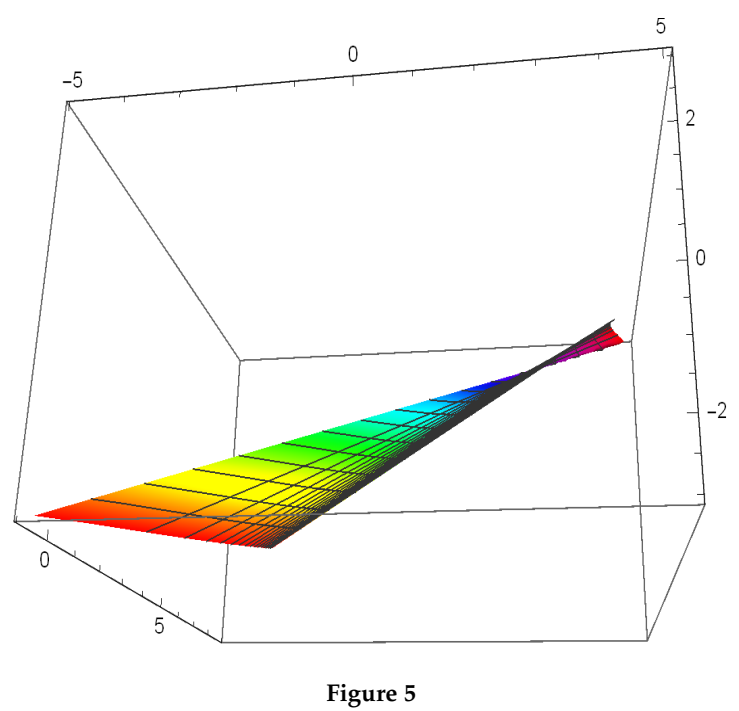

Similar characterizations for ruled surfaces whose base curves are spacelike planar curve (2.9) with zero weighted curvature in $\left(\mathbb{L}^{3}, b y^{2}\right)$ and ruling curves are Smarandache curves of (2.9) can be obtained.

\section{Rotational Surfaces Generated by Non-Null Curves with Zero Weighted Curvature in $\left(\mathbb{L}^{3}, a x^{2}+b y^{2}\right)$}

In this section, we obtain the rotational surfaces generated by spacelike and timelike curves with zero weighted curvature in $\left(\mathbb{L}^{3}, a x^{2}\right)$ and $\left(\mathbb{L}^{3}, b y^{2}\right)$. We get the mean and Gaussian curvatures of these surfaces, give some characterizations for them and draw the graphics of these surfaces. We note that, throughout this section, we are going to take the signs of " \pm " which have been stated in the curves (2.4) and (2.12) as " + ".

Firstly, let us suppose that the spacelike curve (2.4) generates the rotational surfaces. by

If the axis of rotation is spacelike, then from (1.10) and (2.4), the rotational surface in $\mathbb{L}^{3}$ can be parametrized

$$
X(u, v)=\left(x(u) \cosh (v), c_{2}+\int_{1}^{u} \frac{x^{\prime}(t) e^{a x^{2}(t)}}{\sqrt{e^{2 a x^{2}(t)}+c_{1}}} d t, x(u) \sinh (v)\right) .
$$

The normal vector of (4.1) is

$$
\mathcal{N}=-\frac{1}{\sqrt{-c_{1}}}\left(e^{a x^{2}(u)} \cosh (v), \sqrt{e^{2 a x^{2}(u)}+c_{1}}, e^{a x^{2}(u)} \sinh (v)\right) .
$$

Here, $g(\mathcal{N}, \mathcal{N})=\varepsilon=-1$ and so $(4.1)$ is a spacelike surface.

Thus, the Gaussian and mean curvatures of (4.1) are obtained as

$$
K=\frac{2 a e^{2 a x^{2}(u)}}{c_{1}}
$$

and

$$
H=\frac{-e^{a x^{2}(u)}\left(2 a x^{2}(u)+1\right)}{2 \sqrt{-c_{1}} x(u)}
$$

respectively. Hence,

Theorem 4.1. The rotational surface (4.1) generated by spacelike planar curve (2.4) with zero weighted curvature in $\left(\mathbb{L}^{3}, a x^{2}\right)$ is not flat. Furthermore, the Gaussian curvature of the rotational surface (4.1) is positive and negative, if a 0 and $a>0$, respectively.

Theorem 4.2. The rotational surface (4.1) generated by spacelike planar curve (2.4) with zero weighted curvature in $\left(\mathbb{L}^{3}, a x^{2}\right)$ is not minimal but the mean curvature of the rotational surface (4.1) vanishes only for $x(u)=\mp \sqrt{\frac{-1}{2 a}}$. 
We know that, a surface is called a Weingarten surface, if there is a nontrivial relation $\Phi(H, K)=0$, where $H$ is the mean curvature and $K$ is the Gaussian curvature of surface [13]. Hence, from (4.3) and (4.4) we have

Theorem 4.3. The rotational surface (4.1) generated by spacelike planar curve (2.4) with zero weighted curvature in $\left(\mathbb{L}^{3}, a x^{2}\right)$ is a Weingarten surface with the relation

$$
H^{2}=\frac{K\left(1+\ln \left(\frac{c_{1} K}{2 a}\right)\right)^{2}}{4 \ln \left(\frac{c_{1} K}{2 a}\right)} .
$$

Figure 6 shows the rotational surface (4.1) for $x(u)=\sqrt{\ln (u)}, c_{1}=-0.1, c_{2}=4$ and $a=1$.

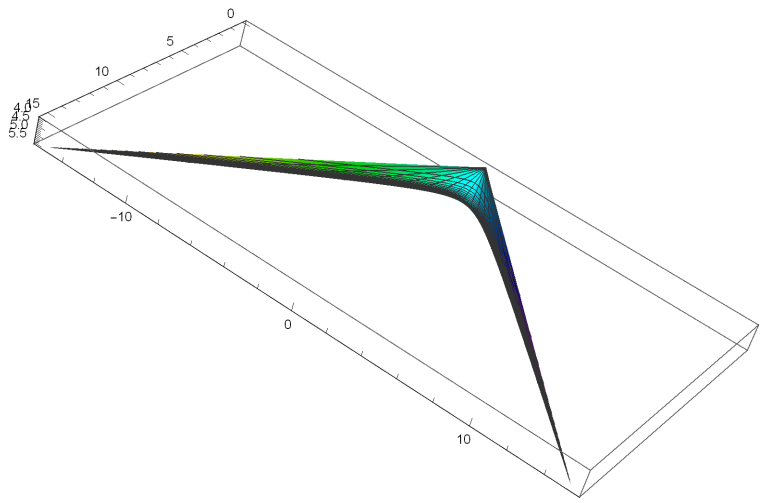

Figure 6

Now, we assume that the axis of rotation is timelike. From (1.10) and (2.4), the rotational surface in $\mathbb{L}^{3}$ can be parametrized by

$$
X(u, v)=\left(x(u),\left(c_{2}+\int_{1}^{u} \frac{x^{\prime}(t) e^{a x^{2}(t)}}{\sqrt{e^{2 a x^{2}(t)}+c_{1}}} d t\right) \cos v,\left(c_{2}+\int_{1}^{u} \frac{x^{\prime}(t) e^{a x^{2}(t)}}{\sqrt{e^{2 a x^{2}(t)}+c_{1}}} d t\right) \sin v\right)
$$

The normal vector of (4.6) is

$$
\mathcal{N}=-\frac{1}{\sqrt{-c_{1}}}\left(e^{a x^{2}(u)}, \sqrt{e^{2 a x^{2}(u)}+c_{1}} \cos (v), \sqrt{e^{2 a x^{2}(u)}+c_{1}} \sin (v)\right) .
$$

Thus, we have $g(\mathcal{N}, \mathcal{N})=\varepsilon=-1$ and so, the surface is spacelike.

Here, the Gaussian and mean curvatures of (4.6) are obtained as

$$
K=\frac{2 a x(u) e^{a x^{2}(u)} \sqrt{e^{2 a x^{2}(u)}+c_{1}}}{c_{1}\left(c_{2}+\int_{1}^{u} \frac{x^{\prime}(t) e^{a x^{2}(t)}}{\sqrt{e^{2 a x^{2}(t)}+c_{1}}} d t\right)}
$$

and

$$
H=-\left(\frac{\sqrt{e^{2 a x^{2}(u)}+c_{1}}+2 a x(u) e^{a x^{2}(u)}\left(c_{2}+\int_{1}^{u} \frac{x^{\prime}(t) e^{a x^{2}(t)}}{\sqrt{e^{2 a x^{2}(t)}+c_{1}}} d t\right)}{2 \sqrt{-c_{1}}\left(c_{2}+\int_{1}^{u} \frac{x^{\prime}(t) e^{a x^{2}(t)}}{\sqrt{e^{2 a x^{2}(t)}+c_{1}}} d t\right)}\right),
$$

respectively.

Theorem 4.4. The rotational surface (4.6) generated by spacelike planar curve (2.4) with zero weighted curvature in $\left(\mathbb{L}^{3}, a x^{2}\right)$ is not flat. Furthermore, the Gaussian curvature of the rotational surface (4.6) is positive and negative, if

$$
a x(u)\left(c_{2}+\int_{1}^{u} \frac{x^{\prime}(t) e^{a x^{2}(t)}}{\sqrt{e^{2 a x^{2}(t)}+c_{1}}} d t\right)<0 \text { and } a x(u)\left(c_{2}+\int_{1}^{u} \frac{x^{\prime}(t) e^{a x^{2}(t)}}{\sqrt{e^{2 a x^{2}(t)}+c_{1}}} d t\right)>0,
$$

respectively. 
Theorem 4.5. The rotational surface (4.6) generated by spacelike planar curve (2.4) with zero weighted curvature in $\left(\mathbb{L}^{3}, a x^{2}\right)$ is not minimal but the mean curvature of the rotational surface (4.6) vanishes only for

$$
1=\frac{-\sqrt{e^{2 a x^{2}(u)}+c_{1}}}{2 a x(u) e^{a x^{2}(u)}\left(c_{2}+\int_{1}^{u} \frac{x^{\prime}(t) e^{a x^{2}(t)}}{\sqrt{e^{2 a x^{2}(t)}+c_{1}}} d t\right)} .
$$

Figure 7 shows the rotational surface (4.6) for $x(u)=\sqrt{\ln (u)}, c_{1}=-0.1, c_{2}=4$ and $a=1$.

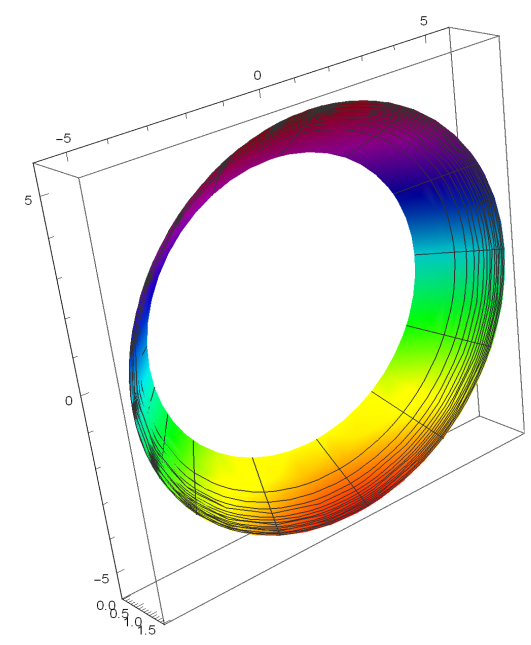

Figure 7

Finally, we assume that the axis of rotation is lightlike. From (1.11) and (2.4), the rotational surface in $\mathbb{L}^{3}$ can be parametrized by

$$
\begin{aligned}
X(u, v)= & \left(\left(1+\frac{v^{2}}{2}\right) x(u)-\frac{v^{2}}{2}\left(c_{2}+\int_{1}^{u} \frac{x^{\prime}(t) e^{a x^{2}(t)}}{\sqrt{e^{2 a x^{2}(t)}+c_{1}}} d t\right)\right. \\
& \frac{v^{2}}{2} x(u)+\left(1-\frac{v^{2}}{2}\right)\left(c_{2}+\int_{1}^{u} \frac{x^{\prime}(t) e^{a x^{2}(t)}}{\sqrt{e^{2 a x^{2}(t)}+c_{1}}} d t\right) \\
& \left.v\left(x(u)-\left(c_{2}+\int_{1}^{u} \frac{x^{\prime}(t) e^{a x^{2}(t)}}{\sqrt{e^{2 a x^{2}(t)}+c_{1}}} d t\right)\right)\right) .
\end{aligned}
$$

The normal vector of $(4.10)$ is

$$
\mathcal{N}=-\frac{1}{2 \sqrt{-c_{1}}}\left(\begin{array}{c}
\sqrt{e^{2 a x^{2}(u)}+c_{1}} v^{2}-e^{a x^{2}(u)}\left(2+v^{2}\right), \sqrt{e^{2 a x^{2}(u)}+c_{1}}\left(v^{2}-2\right)-e^{a x^{2}(u)} v^{2} \\
2\left(\sqrt{e^{2 a x^{2}(u)}+c_{1}}-e^{a x^{2}(u)}\right) v
\end{array}\right) .
$$

From (4.11), we have $g(\mathcal{N}, \mathcal{N})=\varepsilon=-1$; that is, the surface is spacelike.

So, the Gaussian and mean curvatures of (4.10) are obtained as

$$
K=\frac{2 a x(u) e^{a x^{2}(u)}\left(\sqrt{e^{2 a x^{2}(u)}+c_{1}}-e^{a x^{2}(u)}\right)}{c_{1}\left(c_{2}+\int_{1}^{u} \frac{x^{\prime}(t) e^{a x^{2}(t)}}{\sqrt{e^{2 a x^{2}(t)}+c_{1}}} d t-x(u)\right)}
$$

and

$$
H=\left(\frac{\sqrt{e^{2 a x^{2}(u)}+c_{1}}-e^{a x^{2}(u)}+2 a x(u) e^{a x^{2}(u)}\left(c_{2}+\int_{1}^{u} \frac{x^{\prime}(t) e^{a x^{2}(t)}}{\sqrt{e^{2 a x^{2}(t)}+c_{1}}} d t\right)-2 a x^{2}(u) e^{a x^{2}(u)}}{2 \sqrt{-c_{1}}\left(c_{2}+\int_{1}^{u} \frac{x^{\prime}(t) e^{a x^{2}(t)}}{\sqrt{e^{2 a x^{2}(t)}+c_{1}}} d t-x(u)\right)}\right),
$$

respectively. 
Theorem 4.6. The rotational surface (4.10) generated by spacelike planar curve (2.4) with zero weighted curvature in $\left(\mathbb{L}^{3}, a x^{2}\right)$ is not flat. Furthermore, the Gaussian curvature of the rotational surface (4.10) is positive and negative, if

$$
a x(u)\left(c_{2}+\int_{1}^{u} \frac{x^{\prime}(t) e^{a x^{2}(t)}}{\sqrt{e^{2 a x^{2}(t)}+c_{1}}} d t-x(u)\right)>0 \text { and } a x(u)\left(c_{2}+\int_{1}^{u} \frac{x^{\prime}(t) e^{a x^{2}(t)}}{\sqrt{e^{2 a x^{2}(t)}+c_{1}}} d t-x(u)\right)<0,
$$

respectively.

Theorem 4.7. The rotational surface (4.10) generated by spacelike planar curve (2.4) with zero weighted curvature in $\left(\mathbb{L}^{3}, a x^{2}\right)$ is not minimal but the mean curvature of the rotational surface (4.10) vanishes only for

$$
1=\frac{e^{a x^{2}(u)}-\sqrt{e^{2 a x^{2}(u)}+c_{1}}}{2 a x(u) e^{a x^{2}(u)}\left(c_{2}+\int_{1}^{u} \frac{x^{\prime}(t) e^{a x^{2}(t)}}{\sqrt{e^{2 a x^{2}(t)}+c_{1}}} d t-x(u)\right)} .
$$

In Figure 8, one can see the rotational surface (4.10) for $x(u)=\sqrt{\ln (u)}, c_{2}=4, c_{1}=-0.1$ and $a=1$.

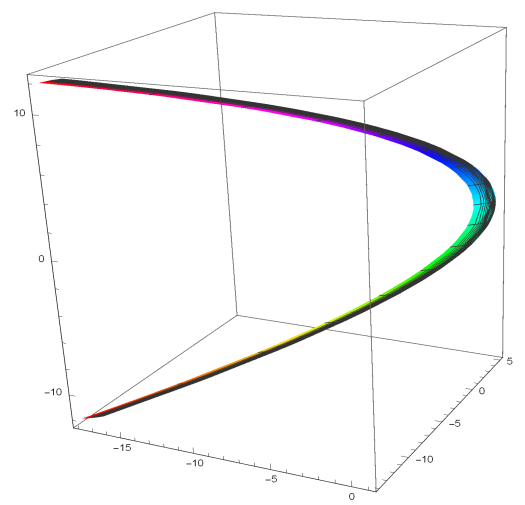

Figure 8

Now, if we suppose that the timelike planar curve (2.13) with zero weighted curvature in $\left(\mathbb{L}^{3}, b y^{2}\right)$ generates the rotational surfaces, then the results can be obtained with the same procedure as above.

Secondly, let us suppose that the timelike curve (2.12) generates the rotational surfaces.

If the axis of rotation is spacelike, then from (1.10) and (2.12), the rotational surface in $\mathbb{L}^{3}$ can be parametrized by

$$
X(u, v)=\left(x(u) \cosh (v), c_{7}+\int_{1}^{u} \frac{x^{\prime}(t)}{\sqrt{c_{8} e^{2 a x^{2}(t)}+1}} d t, x(u) \sinh (v)\right) .
$$

The normal vector of (4.14) is

$$
\mathcal{N}=-\frac{1}{\sqrt{c_{8} e^{2 a x^{2}(u)}}}\left(\cosh (v), \sqrt{c_{8} e^{2 a x^{2}(u)}+1}, \sinh (v)\right) .
$$

Here, $g(\mathcal{N}, \mathcal{N})=\varepsilon=1$ and so (4.14) is a timelike surface.

Thus, the Gaussian and mean curvatures of (4.14) are obtained as

$$
K=\frac{-2 a}{c_{8} e^{2 a x^{2}(u)}}
$$

and

$$
H=\frac{\left(1-2 a x^{2}(u)\right)}{2 x(u) \sqrt{c_{8} e^{2 a x^{2}(u)}}}
$$

respectively. Hence, 
Theorem 4.8. The rotational surface (4.14) generated by timelike planar curve (2.12) with zero weighted curvature in $\left(\mathbb{L}^{3}, a x^{2}\right)$ is not flat. Furthermore, the Gaussian curvature of the rotational surface (4.14) is positive and negative, if a $<0$ and $a>0$, respectively.

Theorem 4.9. The rotational surface (4.14) generated by timelike planar curve (2.12) with zero weighted curvature in $\left(\mathbb{L}^{3}, a x^{2}\right)$ is not minimal but the mean curvature of the rotational surface (4.14) vanishes only for $x(u)=\mp \sqrt{\frac{1}{2 a}}$.

Also, from (4.16) and (4.17) we have

Theorem 4.10. The rotational surface (4.14) generated by timelike planar curve (2.12) with zero weighted curvature in $\left(\mathbb{L}^{3}, a x^{2}\right)$ is a Weingarten surface with the relation

$$
H=\frac{1-\ln \left(\frac{-2 a}{c_{8} K}\right)}{2 \sqrt{\frac{\ln \left(\frac{-2 a}{c_{8} K}\right)}{-K}}} .
$$

Figure 9 shows the rotational surface (4.14) for $x(u)=\sqrt{\ln (u)}, c_{7}=4$ and $c_{8}=a=1$.

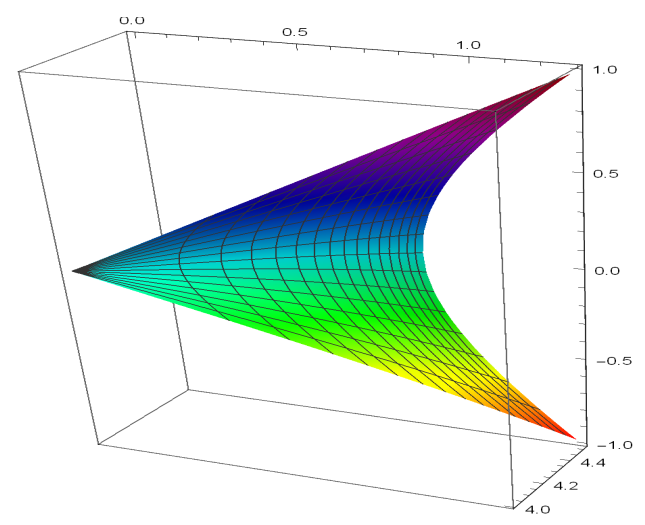

Figure 9

Now, we assume that the axis of rotation is timelike. From (1.10) and (2.12), the rotational surface in $\mathbb{L}^{3}$ can be parametrized by

$$
X(u, v)=\left(x(u),\left(c_{7}+\int_{1}^{u} \frac{x^{\prime}(t)}{\sqrt{c_{8} e^{2 a x^{2}(t)}+1}} d t\right) \cos v, \quad\left(c_{7}+\int_{1}^{u} \frac{x^{\prime}(t)}{\sqrt{c_{8} e^{2 a x^{2}(t)}+1}} d t\right) \sin v\right)
$$

The normal vector of (4.19) is

$$
\mathcal{N}=-\frac{1}{\sqrt{c_{8} e^{a x^{2}(u)}}}\left(1, \sqrt{c_{8} e^{a x^{2}(u)}+1} \cos (v), \sqrt{c_{8} e^{a x^{2}(u)}+1} \sin (v)\right) .
$$

Thus, we have $g(\mathcal{N}, \mathcal{N})=\varepsilon=1$ and so, the surface is timelike.

Here, the Gaussian and mean curvatures of (4.19) are obtained as

$$
K=\frac{-2 a x(u) \sqrt{c_{8} e^{2 a x^{2}(u)}+1}}{c_{8} e^{2 a x^{2}(u)}\left(c_{7}+\int_{1}^{u} \frac{x^{\prime}(t)}{\sqrt{c_{8} e^{2 a x^{2}(t)}+1}} d t\right)}
$$

and

$$
H=\frac{\sqrt{c_{8} e^{2 a x^{2}(u)}+1}-2 a x(u)\left(c_{7}+\int_{1}^{u} \frac{x^{\prime}(t)}{\sqrt{c_{8} e^{2 a x^{2}(t)}+1}} d t\right)}{2 \sqrt{c_{8} e^{2 a x^{2}(t)}}\left(c_{7}+\int_{1}^{u} \frac{x^{\prime}(t)}{\sqrt{c_{8} e^{2 a x^{2}(t)}+1}} d t\right)},
$$

respectively. 
Theorem 4.11. The rotational surface (4.19) generated by timelike planar curve (2.12) with zero weighted curvature in $\left(\mathbb{L}^{3}, a x^{2}\right)$ is not flat. Furthermore, the Gaussian curvature of the rotational surface (4.19) is positive and negative, if

$$
a x(u)\left(c_{7}+\int_{1}^{u} \frac{x^{\prime}(t)}{\sqrt{c_{8} e^{2 a x^{2}(t)}+1}} d t\right)<0 \text { and } a x(u)\left(c_{7}+\int_{1}^{u} \frac{x^{\prime}(t)}{\sqrt{c_{8} e^{2 a x^{2}(t)}+1}} d t\right)>0,
$$

respectively.

Theorem 4.12. The rotational surface (4.19) generated by timelike planar curve (2.12) with zero weighted curvature in $\left(\mathbb{L}^{3}, a x^{2}\right)$ is not minimal but the mean curvature of the rotational surface (4.19) vanishes only for

$$
1=\frac{\sqrt{c_{8} e^{2 a x^{2}(u)}+1}}{2 a x(u)\left(c_{7}+\int_{1}^{u} \frac{x^{\prime}(t)}{\sqrt{c_{8} e^{2 a x^{2}(t)}+1}} d t\right)} .
$$

Figure 10 shows the rotational surface (4.19) for $x(u)=\sqrt{\ln (u)}, c_{7}=4$ and $c_{8}=a=1$.

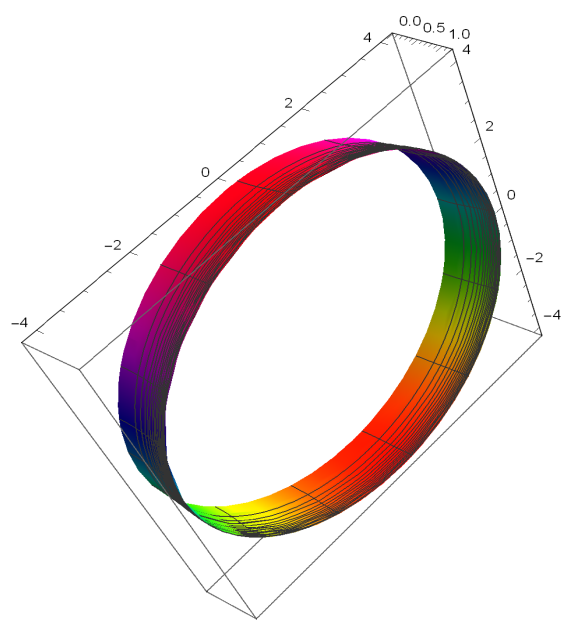

Figure 10

Finally, we assume that the axis of rotation is lightlike. From (1.11) and (2.12), the rotational surface in $\mathbb{L}^{3}$ can be parametrized by

$$
\begin{aligned}
X(u, v)= & \left(\left(1+\frac{v^{2}}{2}\right) x(u)-\frac{v^{2}}{2}\left(c_{7}+\int_{1}^{u} \frac{x^{\prime}(t)}{\sqrt{c_{8} e^{2 a x^{2}(t)}+1}} d t\right),\right. \\
& \frac{v^{2}}{2} x(u)+\left(1-\frac{v^{2}}{2}\right)\left(c_{7}+\int_{1}^{u} \frac{x^{\prime}(t)}{\sqrt{c_{8} e^{2 a x^{2}(t)}+1}} d t\right), \\
& \left.v\left(x(u)-\left(c_{7}+\int_{1}^{u} \frac{x^{\prime}(t)}{\sqrt{c_{8} e^{2 a x^{2}(t)}+1}} d t\right)\right)\right) .
\end{aligned}
$$

The normal vector of $(4.23)$ is

$$
\mathcal{N}=-\frac{1}{2 \sqrt{c_{8} e^{2 a x^{2}(u)}}}\left(\begin{array}{c}
\left(\sqrt{c_{8} e^{2 a x^{2}(u)}+1}-1\right) v^{2}-2, \sqrt{c_{8} e^{2 a x^{2}(u)}+1}\left(v^{2}-2\right)-v^{2}, \\
2 v\left(\sqrt{c_{8} e^{2 a x^{2}(u)}+1}-1\right)
\end{array}\right) .
$$

From (4.24), we have $g(\mathcal{N}, \mathcal{N})=\varepsilon=1$; that is, the surface is timelike.

So, the Gaussian and mean curvatures of (4.23) are obtained as

$$
K=\frac{-2 a x(u)}{\left(\sqrt{c_{8} e^{2 a x^{2}(u)}+1}+1\right)\left(c_{7}+\int_{1}^{u} \frac{x^{\prime}(t)}{\sqrt{c_{8} e^{2 a x^{2}(t)}+1}} d t-x(u)\right)}
$$


and

$$
H=-\left(\frac{\sqrt{c_{8} e^{2 a x^{2}(u)}+1}-1-2 a x(u)\left(c_{7}+\int_{1}^{u} \frac{x^{\prime}(t)}{\sqrt{c_{8} e^{2 a x^{2}(t)}+1}} d t\right)+2 a x^{2}(u)}{2\left(c_{7}+\int_{1}^{u} \frac{x^{\prime}(t)}{\sqrt{c_{8} e^{2 a x^{2}(t)}+1}} d t-x(u)\right) \sqrt{c_{8} e^{2 a x^{2}(u)}}}\right),
$$

respectively.

Theorem 4.13. The rotational surface (4.23) generated by timelike planar curve (2.12) with zero weighted curvature in $\left(\mathbb{L}^{3}, a x^{2}\right)$ is not flat. Furthermore, the Gaussian curvature of the rotational surface (4.23) is positive and negative, if

$$
\operatorname{ax}(u)\left(c_{7}+\int_{1}^{u} \frac{x^{\prime}(t)}{\sqrt{c_{8} e^{2 a x^{2}(t)}+1}} d t-x(u)\right)<0 \text { and } a x(u)\left(c_{7}+\int_{1}^{u} \frac{x^{\prime}(t)}{\sqrt{c_{8} e^{2 a x^{2}(t)}+1}} d t-x(u)\right)>0,
$$

respectively.

Theorem 4.14. The rotational surface (4.23) generated by timelike planar curve (2.12) with zero weighted curvature in $\left(\mathbb{L}^{3}, a x^{2}\right)$ is not minimal but the mean curvature of the rotational surface (4.23) vanishes only for

$$
1=\frac{\sqrt{c_{8} e^{2 a x^{2}(u)}+1}-1}{2 a x(u)\left(c_{7}+\int_{1}^{u} \frac{x^{\prime}(t)}{\sqrt{c_{8} e^{2 a x^{2}(t)}+1}} d t-x(u)\right)} .
$$

In Figure 11, one can see the rotational surface (4.23) for $x(u)=\sqrt{\ln (u)}, c_{7}=4$ and $c_{8}=a=1$.



Now, if we suppose that the spacelike planar curve (2.9) with zero weighted curvature in $\left(\mathbb{L}^{3}, b y^{2}\right)$ generates the rotational surfaces, then the results can be obtained with the same procedure as above.

\section{Acknowledgments}

This paper has been supported by Scientific Research Projects (BAP) unit of İnönü University (Malatya/TURKEY) with the Project number FDK-2018-1349.

\section{References}

[1] Abdel-Aziz, HS., Saad, M.K.: Smarandache Curves Of Some Special Curves in the Galilean 3-Space. Honam Mathematical Journal. 37 (2), 253-264, (2015).

[2] Albujer, A.L., Caballero, M.: Geometric Properties of Surfaces with the Same Mean Curvature in $R^{3}$ and L ${ }^{3}$. J. Math. Anal. Appl. 445, 1013-1024, (2017).

[3] Ali, A.T.: Special Smarandache Curves in the Euclidean Space. Int. J. Math. Comb. 2, 30-36, (2010). 
[4] Ali, A.T.: Position Vectors of curves in the Galilean Space $G_{3}$. Matematnykn Bechnk. 64 (3), 200-210, (2012).

[5] Altın, M., Kazan, A., Karadağ, H.B.: Rotational Surfaces Generated by Planar Curves in E ${ }^{3}$ with Density. Int. J. Anal. Appl., 17 (3), 311-328, (2019).

[6] Altın, M., Kazan, A., Karadağ, H.B.: Ruled Surfaces in $E^{3}$ with Density. Honam Mathematical Journal, 41 (4), 683-695, (2019).

[7] Altın, M., Kazan, A., Karadağ, H.B.: Ruled Surfaces Constructed by Planar Curves in Euclidean 3-Space with Density. Celal Bayar Univ. Journal of Science, 16 (1), 81-88, (2020).

[8] Altın, M., Kazan, A., Karadağ, H.B.: Non-Null Curves with Constant Weighted Curvature in Lorentz-Minkowski Plane with Density. Turk J Math. 44, 588 - 610, (2020).

[9] Baikoussis, C., Blair, D.E.: On the Gauss map of ruled surfaces. Glasgow Math. J. 34, 355-359, (1992).

[10] Belarbi, L. and Belkhelfa, M.: Surfaces in $R^{3}$ with Density. i-manager's Journal on Mathematics. 1 (1), 34-48, (2012).

[11] Choi, J.H., Kim, Y.H., Ali, A.T.: Some associated curves of Frenet non-lightlike curves in E $E_{1}^{3}$. J. Math. Anal. Appl. 394, 712-723, (2012).

[12] Corwin, I., Hoffman, N., Hurder, S., Sesum, V., Xu, Y.: Differential geometry of manifolds with density. Rose-Hulman Und. Math. J. 7 (1), 1-15, (2006).

[13] Dillen, F., Kühnel, W.: Ruled Weingarten surfaces in Minkowski 3-space. Manuscripta Math. 98, 307-320, (1999).

[14] Dillen, F., Pas, J., Verstraelen, L.: On the Gauss map of surfaces of revolution. Bull. Inst. Math. Acad. Sinica. 18, 239-246, (1990).

[15] Divjak, B.: Curves in Pseudo-Galilean Geometry. Annales Univ. Sci. Budapest. 41, 117-128, (1998).

[16] Ekici, C., Öztürk, H.: On Time-Like Ruled Surfaces in Minkowski 3-Space. Universal Journal of Applied Science. 1 (2), 56-63, (2013).

[17] Gromov, M.: Isoperimetry of waists and concentration of maps. Geom. Func. Anal. 13, 178-215, (2003).

[18] Hieu, D.T., Nam, T.L.: The classification of constant weighted curvature curves in the plane with a log-linear density. Commun. Pure Appl. Anal. 13, 1641-1652, (2013).

[19] Kazan, A., Karadağ, H.B.: A Classification of Surfaces of Revolution in Lorentz-Minkowski Space. Int. J. Contemp. Math. Sciences. 6 (39), 1915-1928, (2011).

[20] Kazan, A., Karadağ, H.B.: Weighted Minimal And Weighted Flat Surfaces of Revolution in Galilean 3-Space with Density. Int. J. Anal. Appl. 16 (3), 414-426, (2018).

[21] López, R.: Differential Geometry of Curves and Surfaces in Lorentz-Minkowski space. Int. Electron. J. Geom. 1, 44-107, (2014).

[22] Morgan, F.: Manifolds with Density. Not. Amer. Math. Soc. 52 (8), 853-858, (2005).

[23] Morgan, F.: Myers' Theorem With Density. Kodai Math. J. 29, 455-461, (2006).

[24] Nam, T.L.: Some results on curves in the plane with log-linear density. Asian-European J. of Math. 10 (2), 1-8, (2017).

[25] Şenyurt, S., Altun, Y., Cevahir, C.: Smarandache curves for spherical indicatrix of the Bertrand curves pair. Boletim da Sociedade Paranaense de Matematica. 38 (2), 27-39, (2020), In Press.

[26] Turgut, A., Hacısalihoğlu, H.H.: Timelike Ruled Surfaces in the Minkowski 3-Space-II. Tr. J. of Mathematics. 22, 33-46, (1998).

[27] Turgut, M., Yilmaz, S.: Smarandache Curves in Minkowski Space-time. Int. J. Math. Comb. 3, 51-55, (2008).

[28] Yoon, D.W., Kim, D-S., Kim, Y.H., Lee, J.W.: Constructions of Helicoidal Surfaces in Euclidean Space with Density. Symmetry. 173, 1-9, (2017).

[29] Yoon, D.W.: Weighted Minimal Translation Surfaces in Minkowski 3-space with Density. International Journal of Geometric Methods in Modern Physics. 14 (12), 1-10, (2017).

[30] Yoon, D.W., Yüzbaş1, Z.K.: Weighted Minimal Affine Translation Surfaces in Euclidean Space with Density. International Journal of Geometric Methods in Modern Physics. 15 (11), (2018).

\section{Affiliations}

\section{Mustafa Altin}

AdDrEsS: Bingöl University, Technical Sciences Vocational School, Bingöl-Turkey.

E-MAIL: maltin@bingol.edu.tr

ORCID ID:0000-0001-5544-5910

\section{AHMET KAZAN}

AdDRESS: Malatya Turgut Özal University, Doğanşehir Vahap Küçük Vocational School, Malatya-Turkey.

E-MAIL: ahmet.kazan@ozal.edu.tr

ORCID ID:0000-0002-1959-6102

\section{H.BAYRAM KARADAĞ}

ADDRESS: İnönü University, Faculty of Arts and Sciences, Department of Mathematics, Malatya-Turkey. E-MAIL: bayram.karadag@inonu.edu.tr

ORCID ID:0000-0001-6474-877X 